\title{
Utilization Of Carbon Nanotubes In Electromagnetic Wave Detectors
}

\author{
Muhammad Hanis Zakariah ${ }^{1, a}$, Poppy Puspitasari ${ }^{2}$ \\ ${ }^{l}$ Electrical and Electronic Enginerting Department, Universiti Teknologi PETRONAS \\ 31750 Bandar Seri Iskandar, Tronoh, Perak, Malaysia. \\ ${ }^{2}$ Mechanical Engineering Department, Universitas Negeri Malang, Semarang St. No. 5, East Java, Malang, Indonesia \\ 65144 \\ Corresponding author:*mhanisz@gmail.com
}

\begin{abstract}
Direct detection of hydrocarbon by an active source using electromagnetic (EM) energy termed seabed logging (SBL) has shown very promising results. However, currently available electromagnetic wave technology has a number of challenges include sensitivity and frequency matching. This paper presents development of the carbon nanotubes (CNTs) as electromagnetic wave detector due to outstanding properties of carbon nanotubes. They are currently one of the desired materials for advanced technologies. Two types of detectors were developed in this work, carbon nanotube-based (D1) and without nanotubebased (D2) detectors. Various configuration and arrangement for each type of detector were investigated to determine the one with the highest detection measurement and stability of frequency stability of detection system. It was found that 20 turn-coils coil placed at its centre gives the maximum detection of induction voltage, $39.61 \mathrm{mV}$. However, the 20 turn-coils with CNTs which gives $36.50 \mathrm{mV}$ is the preferred EM detectors due to the stability in frequency of the detection system.

Copyright (C) 2017Journal of Mechanical Engineering Science and Technology
\end{abstract} All rights reserved

Keywords: Sea Bed Logging, Detector, Sensitivity, Frequency.

\section{Introduction}

In SBLs [1], [2], the use of a mobile horizontal electric dipole (HED) source and an array of seafloor electric field receivers are applied. The transmitting dipole emits a low frequency electromagnetic signal which is propagated into the seabed. The array of sea floor receivers measures both the amplitude and the phase of the received signal directly from the transmitter, and waves reflected and guided from the seabed. Received signal depend on the resistivity structure beneath the seabed. Characterisation and detection of the reservoir using SBL are based on the electrical conductivity consisted by all geological media. The principle of differences in of the different conductivity geological media is utilised in this technology[1], [3]. Besides its advantage in segregating a resistivity in a non-conductive layer and conductive formation beneath the sea floor, electromagnetic method has a robustness characteristic against bad operating condition such high temperature and high pressure [4].

Currently available EM wave detectors for the SBL method have a number of setbacks. One of them is the frequency instability which can result in wrong interpretation of the data. These could lead to loss of significant investments.

The work premise deals with utilization of carbon nanotubez as electromagnetic detector. These remarkable materials were chosen due to their tiny structure that leading to extremely high surface area, high chemical stability, high tensile strength and the most important their unique electrical properties. The properties of CNTs depends on their chilarity, which changes the properties from metallic to semi-conducting[5][6][7].

\section{Methodology.}

Carbon nanotube (provided by the University of Cambridge, England) based (D1) and without nanotube-based detectors (D2) were developed in this study to determine their detection ability response to the EM wave. 


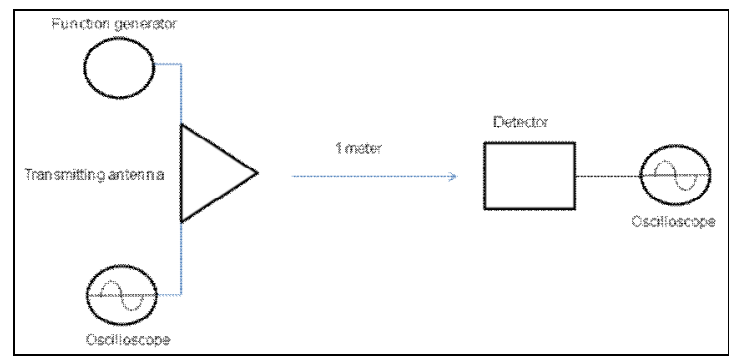

Fig. 1.Block diagram of transmitter-detector system

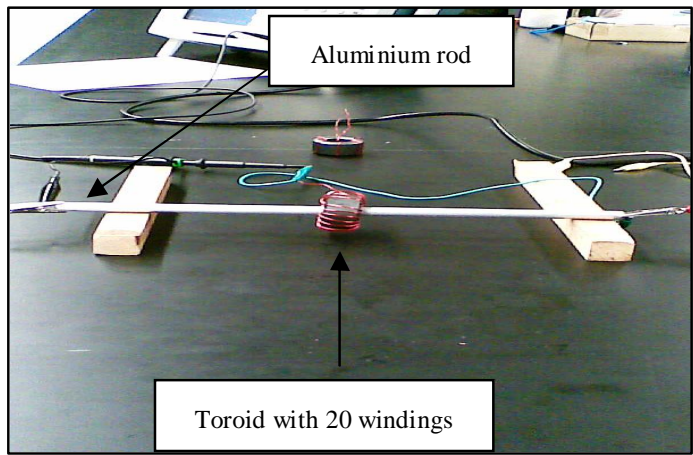

Fig. 2. Transmitter system with CNT and 20 windings copper coils toroid

Table 1. Transmitter properties

\begin{tabular}{cc}
\hline Transmitter parameters & Properties \\
\hline Material & Toroid with 20-turns and aluminium rod in the centre of toroid \\
\hline Frequency & $5 \mathrm{MHz}$ \\
\hline Distance from detector & 1 meter \\
\hline Waveform & Square-shaped \\
\hline
\end{tabular}

Table 2. Detectors main configuration and arrangements

\begin{tabular}{cc}
\hline Detector types & Arrangements \\
\cline { 2 - 2 } Nanotubes (D1) & As conductor \\
\cline { 2 - 2 } & With toroid (probe nanotube) \\
\cline { 2 - 2 } Without Nanotubes (D2) & With toroid (probe at 1-turn toroid) \\
\cline { 2 - 2 } & Toroid with 1-turn copper wire \\
\cline { 2 - 2 } & 20 turn-coils (empty) \\
\hline
\end{tabular}

A 20 turns toroid type transmitter, with an aluminium rod in the centre of toroid is used in this work. It is supplied by $5 \mathrm{MHz}$ frequency of square-shape of waveform from wave generator (Textronic AX493). The distance between the transmitter and the detector fixed at one meter.as described in Figure 1 and Figure 2. The properties of transmitter summarized in Table 1. Two types of EM detectors were investigated. The responses from the detector then were determined by measuring the induced voltage using oscilloscope for all detectors.

Figure 3 shows the arrangement of a carbon nanotube-based detector. Three types of nanotubebased detector (D1) were put into consideration. There are a) nanotube as conductor, b) nanotube with toroid, and c) nanotube with 1-turn toroid and d) nanotubes with 20 turn-coils. Determination of voltage at toroid-based detector (D2) was conducted by using oscilloscope. There were three types of detectors without nanotubes were selected in this work. D2 consists of a) 1-turn toroid, b) 20 turns coil without any conductor placed at the centre of it, and c) 20 turns with aluminium rod at the centre. 


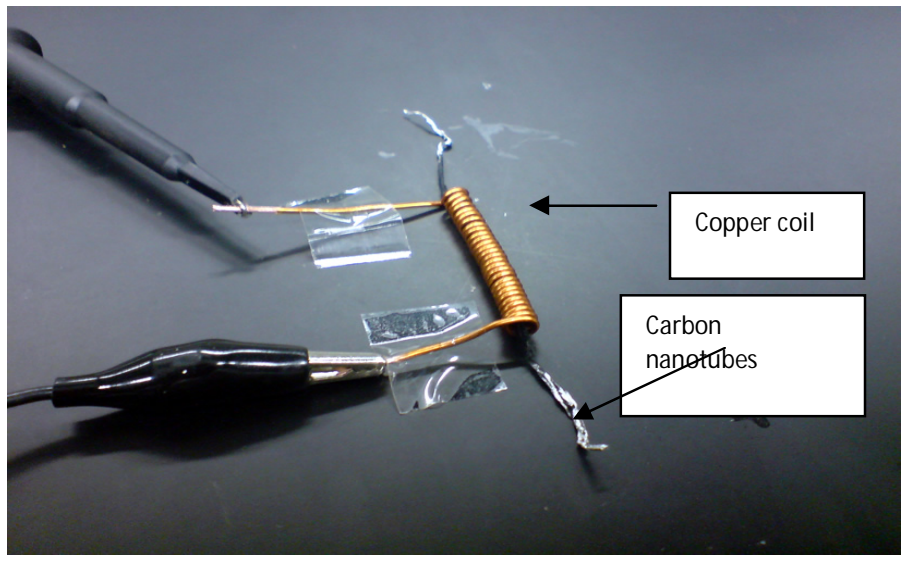

Fig. 3.Coils with nanotubes (Rope) with 20 windings.

\section{Results and discussion.}

Basic ideas to describe how CNTs can detect electromagnetic wave can be found as follows. The CNT-based electromagnetic wave detector operated in a completely different way compared to the traditional electromagnetic wave detector [8]. The detector receives signals via high-frequency mechanical vibrations of the CNTs rather than electrically. These vibrations become significant only when the frequency of the incoming wave matches the resonance frequency of the nanotube. For this reason it allows CNT's gives higher measurement of electromagnetic wave (represents by induced voltage). The simulation studies [9][10] have shown the distribution of electric field surrounding the nanotube radio during the operation of the radio. The field is strongest at the tip of the nanotube and varies as the nanotube vibrates.

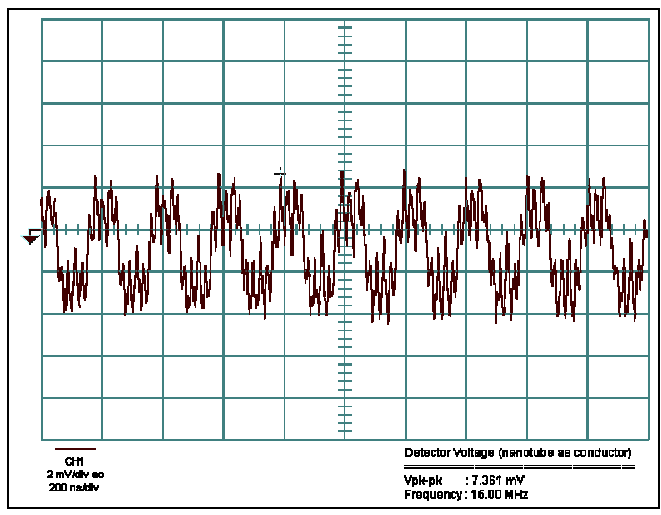

Figure 4 (a). Detector Voltage (nanotubes as conductor).

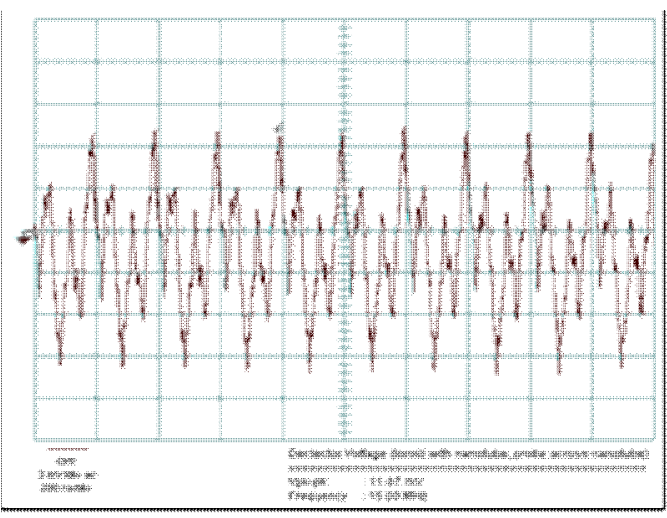

Figure 4 (b). Detector Voltage (toroid with nanotubes, probe across nanotubes). 


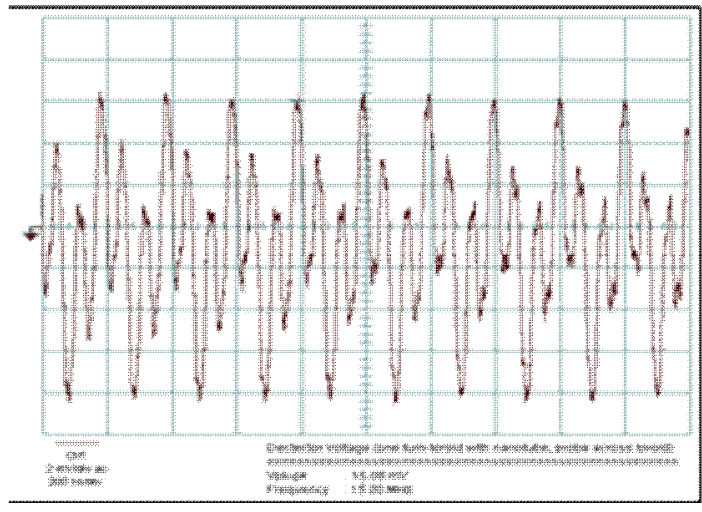

Figure 4 (c). Detector Voltage (one turn-toroid with nanotubes, probe across toroid).

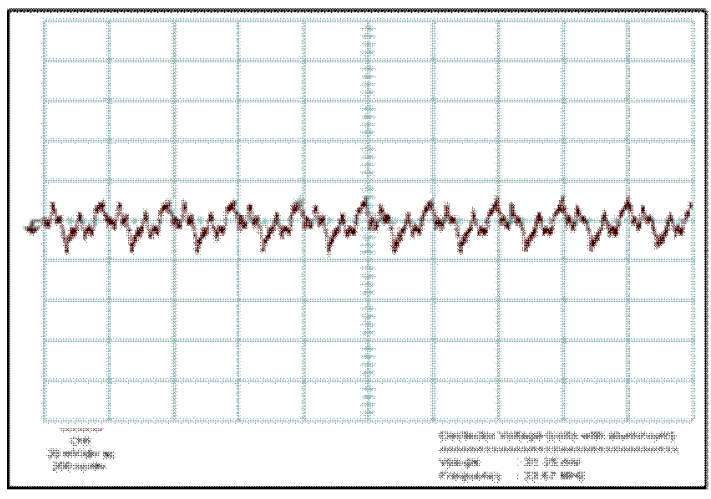

Figure 4 (e). Detector Voltage (coils only)

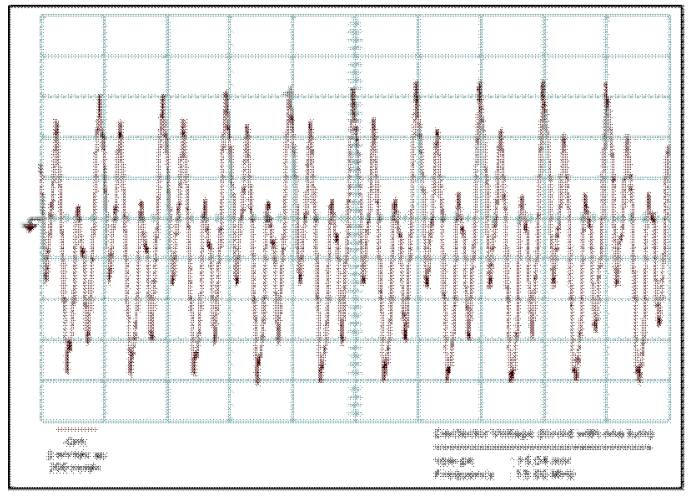

Figure 4 (d). Detector Voltage (toroid with one turn).

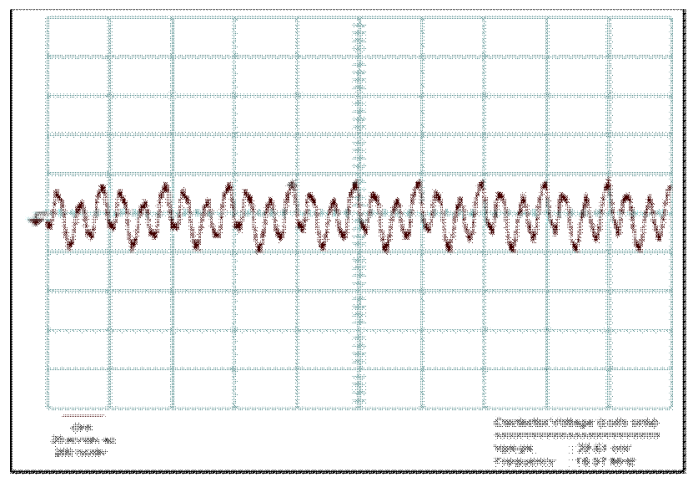

Figure 4 (f). Detector Voltage (coils with aluminum)

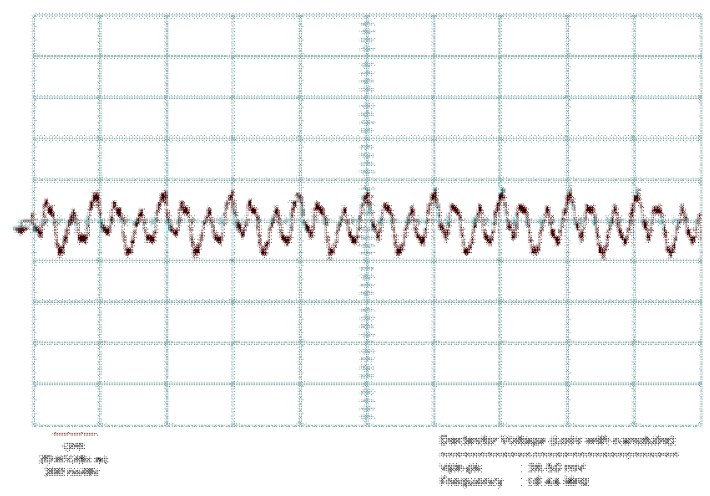

Figure 4 (g). Detector Voltage (coils with nanotubes).

Fig. 4.(a) to (g) shows the Vp-p in detectors for all the 7 types of detector listed in Table 1.

The Vp-p values of the detectors are summarized in Table 3. It was shown that, by using a D1(a), (b), and (c), and (d)Vp-p obtained from the oscilloscope were $7.361 \mathrm{mV}, 11.87 \mathrm{mV}$ and $15.080 \mathrm{mV}$ and $36.50 \mathrm{mV}$ respectively. Voltage values measured from D2 (a), (b), and (c) were $15.04 \mathrm{mV}$, $39.61 \mathrm{mV}, 31.25 \mathrm{mV}$ 
Table 3. $\mathrm{V}_{\mathrm{p}-\mathrm{p}}$ and frequency values obtained from detectors.

\begin{tabular}{cccc}
\hline Detector configuration & $\mathbf{V}_{\mathbf{p}-\mathbf{p}}(\mathbf{m V})$ & Frequency $(\mathbf{M H z})$ & Figure \\
\hline Nanotubes as conductor & 7.36 & 15.00 & $4(\mathrm{a})$ \\
\hline Nanotubes with toroid (probe nanotubes) & 11.87 & 15.00 & $4(\mathrm{~b})$ \\
\hline Nanotubes with toroid (probe 1-turn toroid) & 15.08 & 15.08 & $4(\mathrm{c})$ \\
\hline 1-turn toroid & 15.04 & 15.08 & $4(\mathrm{~d})$ \\
\hline 20 turn-coils (empty) & 39.61 & 16.87 & $4(\mathrm{e})$ \\
\hline 20 turn-coils with aluminium & 31.25 & 33.67 & $4(\mathrm{f})$ \\
\hline 20 turn-coils with nanotubes & 36.50 & 18.44 & $4(\mathrm{~g})$ \\
\hline
\end{tabular}

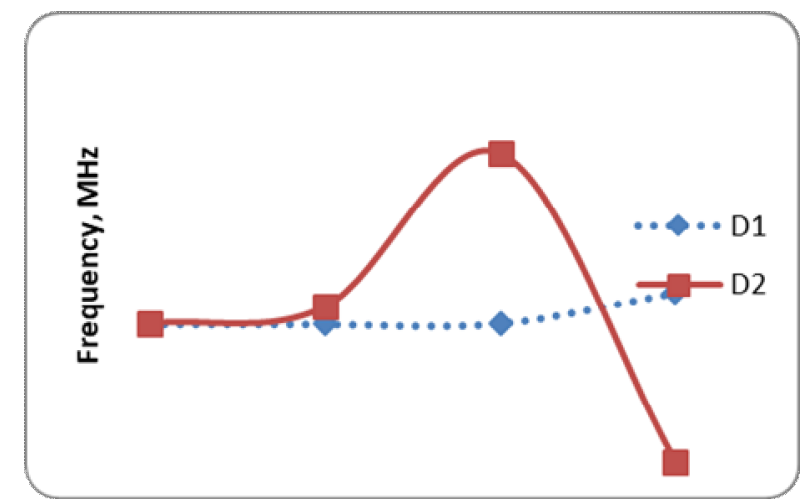

Figure 5. Detectors frequency comparison between D1 and D2

Noted above, to measures electromagnetic wave, vibrations in the CNT become a plays a major role [11]-[13]. Detection of the highest sensitivity of electromagnetic wave can be obtained when the frequency of the incoming wave matches the resonance frequency of the CNT. Figure 5 shows a comparison between frequeny obtained at the D1 and D2. Frequency obtained at the detectors is a critical aspect need to be consider to determine wheather D1 or D2 have a stability in frequency matching.

Values of detector's frequency should clearly clarified. It can lead to the determination of resonance in the detector to give the accurate measurements of the incoming electromagnetic wave [3], [8]. In this work, we choosed which detectors gave the most suitable reading of the frequency obtained at the oscilloscope. In this initial work it was found frequency at D1(CNT) was more stable compared with D2 (Table 3)

\section{Conclusion.}

From the results table shown in Table 2 it was observed the air filled 20 turn-coils gave the maximum detection of $39.61 \mathrm{mV}$. However, the 20 turns nanotubes which gives $36.60 \mathrm{mV}$ is the preferred EM detectors due to the frequency stability of detection system. It should be noted that nanotubes as EM detectors could be improved by placing it in the centre of a ferrite toroid. Nanoparticle ferrite can also be encapsulated in the nanotubes to give detection.

\section{Future work}

We hope to construct prototype of CNT-based electromagnetic wave detector with a tuner to receive only a preselected band of electromagnetic wave frequency.

\section{Acknowledgement}

The author wishes to thank the SBL group of Universiti Teknologi PETRONAS for providing the facilities and all colleagues who previously provided technical support. We appreciate ACME Ferrite Products Sdn. Bhd who give a support in provides Ferrite products that been used in this experiment. 


\section{References}

[1] T. Eidesmo et al., "Sea Bed Logging (SBL), a new method for remote and direct identification of hydrocarbon filled layers in deepwater areas," First Break, vol. 20, no. 3, pp. 144-152, 2002.

[2] H. Carstens, "The same PRINCIPLE as in borehole logging."

[3] S. E. Johnstad, B. A. Farrelly, and C. Ringstad, "Seabed Logging On The North Sea Troll Field," Offshore Technol. Conf., pp. 1-3, 2005.

[4] D. A. Toffelmier and J. A. Tyburczy, "Electromagnetic detection of a 410-km-deep melt layer in the southwestern United States," Nature, vol. 447, no. 7147, pp. 991-994, 2007.

[5] V. N. Popov, "Carbon nanotubes: Properties and application," Materials Science and Engineering R: Reports, vol. 43, no. 3. pp. 61-102, 2004.

[6] C. Rutherglen and P. Burke, "Carbon nanotube radio,” Nano Lett., vol. 7, no. 11, pp. 3296-3299, 2007.

[7] E. Properties, "Carbon Nanotubes : An Overview Nanotubes and the future," 1800.

[8] H. Daud, N. Yahya, V. Sagayan, and M. Talib, “A scaled experiment 2 for verification of SPLINE Interpolation Technique for sea bed logging method," in Proceedings - 2011 IEEE International Conference on Control System, Computing and Engineering, ICCSCE 2011, 2011, pp. 80-85.

[9] M. Niaz, N. Yahya, K. Koziol, and N. Nasir, "Composites for Their Application in Sea Bed Logging," Ceram. Int., vol. 37, pp. 3237-3245, 2011.

[10] J. T. Lucy MacGregor, "Marine controlled-source electromagnetic methods in the hydrocarbon industry: A tutorial on method and practice," GeoScienceWorld, vol. 2, no. 3, 2014.

[11]E. T. Thostenson, Z. Ren, and T.-W. Chou, "Advances in the science and technology of carbon nanotubes and their composites: a review," Compos. Sci. Technol., vol. 61, no. 13, pp. 1899-1912, 2001.

[12] Y.-P. Sun, K. Fu, Y. Lin, and W. Huang, "Functionalized Carbon Nanotubes: Properties and Applications," Acc. Chem. Res., vol. 35, no. 12, pp. 1096-1104, 2002.

[13] G. A. Rivas et al., "Carbon nanotubes for electrochemical biosensing," Talanta, vol. 74, no. 3. pp. 291307, 2007. 